\title{
Eph and Ephrin Signaling in Mammary Gland Morphogenesis and Cancer
}

\author{
Anne-Catherine Andres ${ }^{1}$ and Andrew Ziemiecki ${ }^{1,2}$
}

\begin{abstract}
The Eph family of receptor tyrosine kinases and their membrane-bound ligands, the ephrins, play a central role in pattern formation during embryonic development and there is growing evidence that they are also instrumental in the control of tissue dynamics in the adult. The mammary gland is a paradigm for morphogenic processes occurring in the adult, since the gland develops predominantly postnatally and is subjected to continuous cyclic remodeling according to functional demands. Thus, pattern formation and the establishment of a functional organ structure are permanent themes in the mammary gland life cycle. In this paper we summarize the experimental evidence and discuss possible mechanisms by which Ephs and ephrins are modulating mammary epithelial cell adhesion, communication, and migration. Furthermore, we speculate on the different aspects of their influence on normal mammary gland development, function, and carcinogenesis.
\end{abstract}

KEY WORDS: Mammary gland development; mammary epithelial cells; mammary stem cells; branching morphogenesis; lobulo-alveolar development; cell adhesion; cell-cell communication; cell-matrix interaction; pattern formation; body plan; mammary endothelium; vascularization.

The proper development and functioning of the mammary gland is strictly dependent on a complex interplay of endo- and paracrine stimulation, cell-cell communication, and cell-matrix interactions. In contrast to most organs, these morphogenic processes are not limited to the embryonic period but occur mainly postnatally. Thus, the mammary gland represents a unique model to study developmental mechanisms normally limited to the embryonic period. Studies in many laboratories during the past 10 years have revealed that the Eph receptor tyrosine kinase and ephrin ligand families, by influencing cell adhesion and/or migration, are effectors of many developmental processes, including segmentation, pattern formation, cell boundary formation, and cell guidance during embryogenesis. In this paper we focus on the involvement of these receptor-ligand families in the postnatal morphogenesis of the mammary gland.

\footnotetext{
${ }^{1}$ The Tiefenau Laboratory, Department of Clinical Research, University of Bern, Bern, Switzerland.

${ }^{2}$ To whom correspondence should be addressed at Department of Clinical Research, Tiefenaustrasse 120, CH-3004 Bern, Switzerland; e-mail: andrew.ziemiecki@dkf3.unibe.ch.
}

\section{THE Eph AND EPHRIN FAMILIES}

The Eph receptor family, with 14 characterized members in mammals to date, represents the largest family of RPTKs. The extracellular moiety of these molecules consists of an immunoglobulinlike globular domain involved in ligand interaction, a cysteine-rich region and two fibronectin type III repeats. A highly conserved motif, YIDPFTYEDP, found in the internal juxtamembrane region of all Eph family members participates by specific protein interactions in downstream signaling complex formation and in the regulation of the catalytic and biological activity of the receptors. The kinase domain is located intracellularly together with a conserved SAM thought to represent a protein-binding module involved in developmental regulation. It is

Abbreviations used: FAK, focal adhesion kinase; GPI, glycosylphosphatidylinositol; LTR, long terminal repeat; MMTV, mouse mammary tumor virus; PDZ, postsynaptic density protein, disc large, zona occludens; RPTK, receptor tyrosine kinase; SAM, sterile alpha motif; SH2, Src homology domain-2; SH3, src homology domain-3. 
speculated that the SAM motif participates in the formation of heterodimers, thereby regulating receptor oligomerization and activation. The C-termini of Eph RPTKs have consensus PDZ binding motifs, which can bind PDZ domain-containing proteins, many of which are implicated in the formation of submembranous scaffolds for the assembly of macromolecular signaling complexes (Fig. 1(A)) $(1,2)$.

The protein ligands of the Eph family, the ephrins, are also membrane-associated, either by a glycosy-phosphatidylinositol (GPI) anchor (ephrinA family) or as transmembrane proteins (ephrinB family). Extracellularly, the molecules consist of the receptor binding domain containing a high affinity receptor binding loop and a lower affinity interface, probably mediating the formation of tetrameric structures (Fig. 1(B)), (3). The intracellular moiety of ephrin-B family members contains five tyrosine residues conserved in location and amino acid context. In addition, a PDZ binding motif is located at the C-terminus of ephrin-B family members and has been shown to interact with PDZ domain-containing proteins (Fig. 1(A)) $(1,2)$.

Although receptor-ligand interaction preferences have been observed, both receptors and ligands are promiscuous and can interact with several partners, albeit with different affinities. In general, EphA receptors interact with ephrin-A ligands, whereas EphB receptors preferentially bind to ephrin-B ligands. Given the membrane-bound localization of both receptors and ligands, it is not surprising that ligands have been reported to be most effective in receptor activation when cell surface-bound and clustered. The cytoplasmatic domain of the ephrin ligands undergoes phosphorylation on conserved tyrosine residue(s) following receptor interaction in vitro and in vivo $(1,2)$. Thus, SH2-driven signaling cascades can ensue not only from the receptors but also from the ligands, provoking bidirectional signaling and mutual cell-cell communication (Fig. 1(B)). Possible mechanism(s) for modulating both receptor and ligand activities exist. Both molecule types have been shown to associate with phosphatases $(1,2)$, and, recently, an extracellular metalloprotease, Adam 10, has been found to associate with ephrin-A ligands. This protease is activated following receptor engagement and cleaves the extracellular moiety of the ligand molecule, terminating ligand signaling and the physical association between cells (4).

Intracellularly, a wide range of molecules has been implicated in the propagation of Eph and ephrin-derived signals, including $\mathrm{SH} 2$ and $\mathrm{SH} 3$

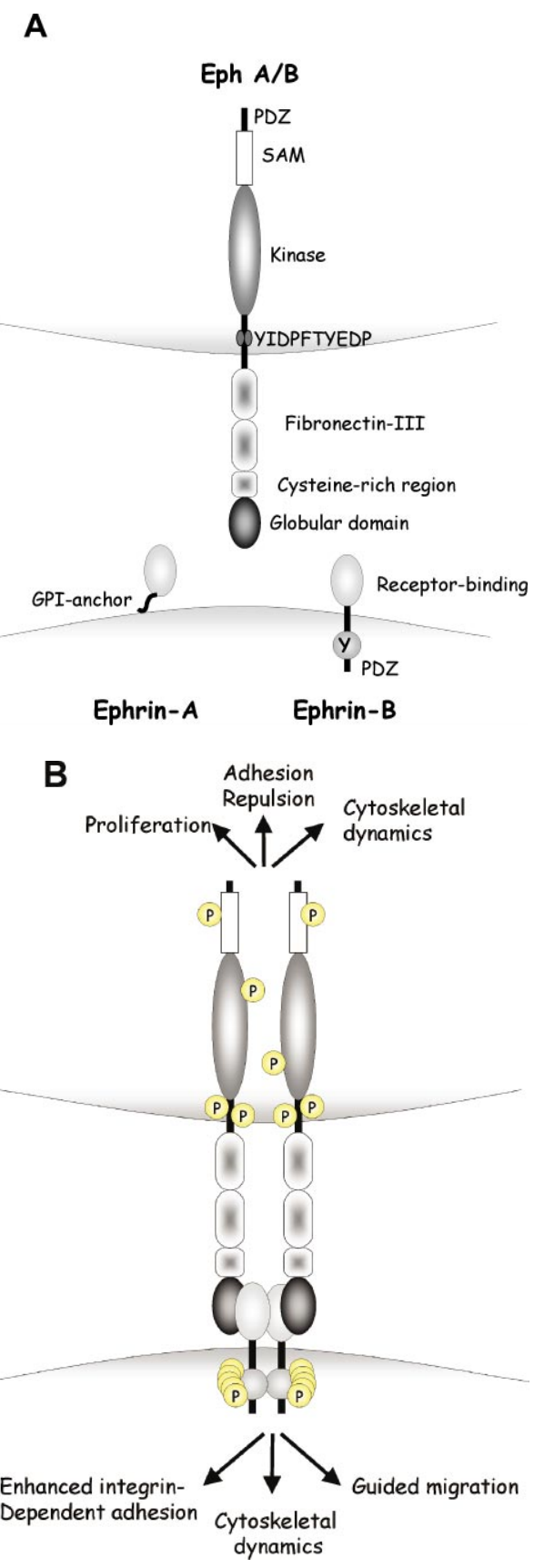

Fig. 1. (A) Schematic representation of the structure of the Eph receptors and ephrin ligands. The A-type ephrins (membrane bound via GPI-tail) and B-type ephrins (transmembrane molecules with conserved intracellular tyrosine residues) are structurally distinct. A- and B-type receptors are structurally similar and are grouped according to their preferences in ligand interaction. (B) Schematic representation of the interaction between $\mathrm{EphB}$ receptors and ephrin-B ligands. Receptor-ligand interaction leads to the dimerization and concomitant tyrosine phosphorylation of both receptor and ligand. Signaling cascades can ensue from both receptor and ligand, provoking a plurality of responses affecting cell shape, adhesion, and migration. 
Table I. List of Molecules Known to Either Interact or Communicate With Eph Receptors and Ephrin Ligands (for Review See Ref. 1)

\begin{tabular}{lll}
\hline & \multicolumn{1}{c}{ Interacting molecules } & \multicolumn{1}{c}{ Cross-talk } \\
\hline Eph & Ephs & Cadherins \\
& Ephrins & Integrins \\
& Rho and Ras family of small GTPases & Wnt signaling pathway \\
& Exchange factors ephexin, intersectin, kalirin & Ryk \\
& Adaptor proteins Crk, Grb7, Grb2, Grb10, Shc, Nck, Cbl & NMDA receptors \\
& Docking protein Cas & T-cell receptor \\
& Kinases FAK, src, abl & \\
Phosohatases Shp2, LMW-PTP & \\
& PI 3-kinase & \\
& Ubiquitin ligase & \\
Ephs & Cadherins \\
Ephrins & Integrins \\
& GTPase-activating protein PDZ-RGS3 & Wnt signaling pathway \\
& Kinases FAK, src, fyn & FGF-receptor \\
& Adaptor protein Grb4 & PDGF-receptor \\
& Phosphatase PTP-BL & Tie-2 \\
\hline
\end{tabular}

domain-containing kinases and adapter proteins, PDZ domain-binding proteins and the Rho, Rac, and Ras family of GTPases $(1,2)$ (Table I). Although the plurality of effects observed upon Eph and ephrin stimulation is large, a common theme appears to be the modulation of adhesive and/or migratory properties of cells by targeting the cytoskeleton (Fig. 1(B)) (5).

The observation that Eph receptors and their ligands exhibit reciprocal expression patterns during embryonic development has led to the suggestion that these molecules play a role in the development and patterning of a variety of tissues during embryogenesis. Indeed, Eph and ephrin family members have been shown to be involved in gastrulation, cell migration from the neural crest, segmentation of the early embryo, and formation of the somites. In the developing nervous system, they play a pivotal role in axon guidance and fasciculation. Furthermore, these molecules are involved in cell sorting and restriction of intermingling and communication resulting in tissue boundaries $(6,7)$. EphB2, -B3 and -B4 and ephrin$\mathrm{B} 1$ and -B2 also participate in the adhesive response of endothelial cells during embryogenesis. Ephrin-B2 is exclusively expressed in embryonic arteries, whereas EphB4 marks the embryonic veins. Disruption of either gene causes embryonic lethality by E10.0 and is accompanied by defects in early vascular development and myocardial trabeculation in the heart (8).

In contrast to embryonic development, little is known about the function(s) of the Eph and ephrin families in postnatal and adult life. Some members are expressed in adult organs, such as mammary gland, kidney, and lung (9), and ephrin-B2 expression has been demonstrated in adult arteries of varied diameter, in subsets of microvessels at sites of adult neovascularization and within experimental tumor vasculature. Furthermore, ephrin-B2 expression has also been observed in vascular smooth muscles of arteries but not veins $(10,11)$. These observations suggest that members of the Eph and ephrin families are also instrumental in inductive cell movement, structure formation, and homeostasis in the adult.

In conclusion, Ephs and ephrins are multifunctional molecules governing the control of proliferation and death, cellular shape, cell adhesion, and repulsion. They exhibit a complex mechanism of activation and action depending on the environment. Signaling can occur either unidirectionally or bidirectionally and ligand-receptor interactions are highly promiscuous. Furthermore, receptor activation can occur in the absence of ligand and receptor and/or ligand activation can also be achieved by cross-talk with other cell signaling pathways $(1,2,7)$ (Table I).

\section{Eph AND EPHRIN EXPRESSION IN THE MAMMARY GLAND}

Although a systematic survey of Eph and ephrin expression in the mammary gland is lacking, several studies have shown that at least some family members are differentially expressed in this organ. The EphB4 receptor, originally isolated from mouse mammary epithelial cells (9), is expressed in myoepithelial, 
luminal, and ductal epithelial cells predominantly during puberty and the follicular phase of the estrus cycle in mice and humans $(9,12,13)$. Estrogen is one of the mediators conferring this tight control of expression. Ovariectomized mice fail to express EphB4, and administration of estrogen can restore expression (12). Estrogen control is likely to occur at both the transcriptional and posttranscriptional levels. Although estrogen-responsive elements are found in the promoter region of both the EphB4 and ephrin-B2 genes (unpublished observation), the estrogen effects are most evident at the protein level, a phenomenon also observed for other Eph genes $(12,14)$. Interestingly, the opposite effect of estrogen on EphB4 expression has been documented in the glandular epithelium of the endometrium, suggesting that EphB4 is either differentially controlled by the different estrogen receptors or that cell-type specific conversion of the estrogen signal may lead to the differential response (15). In addition to EphB4, developmentally controlled expression of EphA2 in the mammary epithelium has also been reported $(9,16)$. Furthermore, in vitro studies have shown that EphA2 expression is negatively regulated by estrogen and $c-m y c$ (17). The cognate EphB4 ligand, ephrin-B2, shows a similar cell-type specific expression in the mammary gland, however, with less strict hormonal control (12).

In addition to estrogen, transcription factors of the Tcf family may also contribute to the specific expression of Eph and ephrins in the mammary gland. Members of this family of transcription factors have been implicated in the establishment of epithelial cell fate predominantly in intestine, skin, and the mammary gland (18). In the intestine, Tcf has been shown to simultaneously stimulate the expression of EphB receptors and repress transcription of ephrin-B ligands (19).

\section{Eph AND EPHRIN INVOLVEMENT IN MAMMARY GLAND MORPHOGENESIS}

The morphogenesis of the mammary gland is accomplished by locally regulated cell proliferation and pattern formation involving controlled bifurcations of the growing ducts and the development of functional, secretory lobulo-alveolar units. At birth, a modestly ramifying mammary ductal system extends under the skin in a small mass of adipose tissue. With the onset of ovarian function, the epithelial ducts elongate by massive directional growth of the end-buds, which invade the fatty stroma and by twisting and branching give rise to the adult ductal network capable of growth and differentiation during each pregnancy. Eph and ephrins are instrumental in cell guidance during embryonic development. Depending on the microenvironment and the identity of interacting partners, Eph and ephrin activation can exert cellular repulsion, adhesion, or attraction (20). The molecular mechanisms responsible for the cyclic pattern formation of the mammary ductal tree are largely unknown. However, emerging experimental evidence implicates members of the Eph and ephrin families either directly or via cross-talk with other signaling pathways in the control of mammary morphogenesis.

\section{Eph/Ephrin Cross-Talk With Cell Adhesion Complexes}

The dynamic structure of the mammary gland depends on the ability to form and disassemble cell-cell contacts. There is compelling evidence that Eph and ephrin are controlled by or are controlling cell-cell adhesion complexes. A major component of cell adhesion complexes are the cadherins, which play an important role in the establishment of the mammary ductal tree. The bilayered structure of the mammary epithelial duct is established in the end-bud, where the cap cells comprise a monolayer of stem cells overlying a population of cells giving rise to the luminal epithelial cells. Cap cells develop either into myoepithelial cells or migrate into the luminal compartment to constitute the stem cell population (21). It has been shown that P-cadherin on cap cells and E-cadherin on the luminal cells are instrumental in the organization of these two compartments, and E-cadherin-mediated cell contacts are a prerequisite for the formation of a functional secretory epithelium (22). Interestingly, Eph and ephrin expression can be modulated by Ecadherin. It has been shown in embryonic stem cells that disruption of E-cadherin expression leads to the induction of the EphB2 and -B4 receptors and ephrin$\mathrm{B} 1$ and -B2 ligands. In contrast, loss of E-cadherin expression resulted in the loss of the expression of EphA receptors (23). Moreover, membrane localization and phosphorylation of EphA2 is dependent on E-cadherin-mediated cell adhesion in breast epithelial cells (24). In light of these observations, it is tempting to speculate that EphA receptors are predominantly involved in anchoring epithelial cells, whereas EphB receptors and ephrin-B ligands are instrumental in guided cell migration. Applied to the mammary gland, Eph and ephrins may participate in the cadherin-mediated compartmentalization of the 
mammary epithelial cells, with type A signaling supporting luminal cell adhesion and type B signaling determining epithelial outgrowth and migration.

In addition to the cadherins, AF6 protein is a component of adherens and tight junctions, and its absence correlates with disruption of epithelial cellcell junctions and cell polarity during mouse development (25). AF6 has been shown to interact directly with several members of the Eph family via its PDZ domain; in the case of EphB3 this interaction is dependent on receptor activation (26). In addition, AF6 is a target for the Ras family of proteins (27). In this context, the interactions of Ephs with the Ryk receptor are of particular interest. Ryk is an orphan receptor with no intrinsic catalytic activity. It is expressed predominantly during embryonic development, but also in the adult mammary epithelium (28-30). Murine EphB2 and EphB3 interact with Ryk, leading to its activation by phosphorylation and provoking the association of these three proteins with AF6, thereby stabilizing junctional complexes (31). Interestingly, $R y k^{-}$mice have a cleft palate, a comparable phenotype as observed in EphB2/EphB3 double knockout mice, supporting the postulated cooperation between these two molecules in morphogenic processes (32). These observations strongly implicate Ephs and ephrins as potential modulators of cell-cell contacts at different levels of cell junction signaling

\section{Eph/Ephrin Cross-Talk With Focal Adhesion Complexes}

In addition to cell-cell communication, interactions with the extracellular matrix are necessary for the proper development of the mammary gland. Correct functioning of the integrins is essential for the shaping of mammary epithelial cells and for their secretory activity (33) (for more details see articles by Glukhova and Streuli in this issue). Both Eph receptors and ephrin ligands have been shown to interact and cross-talk with the integrin signaling cascade at different levels, implicating them in an additional aspect of mammary gland function. EphA receptors have been reported to suppress integrin signaling, whereas a promoting effect has been ascribed to EphB receptors (34-37). This crosstalk can occur at different molecular levels: via dissociation and redistribution of focal adhesion complex components such as FAK and paxillin (35), via phosphorylation and modulation of the activities of the small GTPases R-ras and Rap1 (36) and via transient or constitutive interactions with PI-3 kinase (38-40). Similarly, the ephrins are capa- ble of modulating cell adhesion by increasing FAK activity, redistributing paxilin and disassembly of focal adhesions (41). In conclusion, Eph and ephrins seem to be involved in the fine tuning of basement membrane anchoring and detachment, an aspect of particular importance during the dynamic states of mammary gland development. Indeed, expression of Eph and ephrins is predominantly observed during puberty and the estrous cycle and is downregulated during the differentiated, rather static state at endpregnancy and lactation $(9,12)$.

\section{Eph/Ephrin Cross-Talk With Wnt Signaling}

As already mentioned, Eph/ephrin expression can be modulated by members of the Tcf transcription factor family. The activity of Tcf is dependent on the availability of cytoplasmic free $\beta$-catenin which in turn is controlled by the Wnt signaling pathway (42). Members of the Wnt family are differentially expressed during mammary gland development, and Wnt-4 signaling has been shown to be essential in mediating progesterone function during mammary gland morphogenesis $(43,44)$ (for more details see paper by A. Clarke in this issue). These observations implicate direct cross-talk between the Eph/ephrin and Wnt signaling pathways during mammary gland development and functioning.

A potentially important implication of this crosstalk in mammary gland biology may concern the control of stem cell fate. The capacity of the mammary gland to undergo cyclic functional development upon demand is thought to originate from multipotent stem cells scattered along the ductal tree which are capable of self-renewal and differentiation into the various types of epithelial cells present in the lactating mammary gland (45). There is presently little experimental information available how the distribution, maintenance, regeneration, and differentiation of the mammary stem cells are regulated at the molecular level. The Wnt signaling cascade has been implicated in the maintenance of stem cell populations in various organ systems; however, the mechanisms by which Wnt sustains stem cell populations remain unclear (46). The recent observations made in intestinal villi may serve as a possible model for mammary regeneration. It has been shown that the $\beta$-catenin/Tcf- regulated expression of EphB2 and EphB3 and ephrin-B1 and -B2 demarcate the proliferative stem cell population from the differentiating cells, respectively (19). The molecular mechanisms regulating proliferation versus differentiation 
involve pathways modulating cell adhesiveness and migration, notably the Rac/Rho/Ras GTPases and the MAP kinase pathway, key intracellular targets of Eph-ephrin signaling $(1,2)$. As mentioned above, selective expression of cadherins, in addition to $\mathrm{Wnt}$, are involved in the formation of the mammary stem cell population. Bearing in mind that Eph and ephrins participate in cross-talk with both families of molecules, it is tempting to speculate that they are also involved in the regulation of distribution, proliferative activity, and fate of the mammary epithelial stem cells. The dissection of the molecular pathways, however, is hampered by the fact that, in contrast to the welldemarcated location of stem cells in the gut, the mammary stem cells are randomly scattered in the epithelial ducts, and well-defined molecular stem cell markers are lacking.

\section{EXPERIMENTAL EVIDENCE FOR A ROLE OF Eph AND EPHRIN IN MAMMARY GLAND BIOLOGY}

To date, direct experimental evidence for the involvement of members of the Eph/ephrin families in the development of the mammary gland is limited to studies of transgenic mice expressing the EphB4 receptor gene under the control of the MMTV-LTR promoter (47). These transgenic mice exhibit strong
EphB4 expression in the mammary epithelium during pregnancy and lactation, developmental stages at which the expression of the endogenous gene is downregulated. The phenotypic consequences observed in the mammary glands of these mice include delayed epithelial proliferation during pregnancy and suppression of epithelial cell death at the onset of postlactational involution. Furthermore, the mammary parenchyma is characterized by the development of fewer side-branches during pregnancy which, however, are composed of more but smaller lobulo-alveolar units than those of control mice. At lactation, the secretory alveolar cells exhibit a highly irregular morphology, with many epithelial cells exfoliating into the lumen. Moreover, the regional absence of milk fat globules indicates disturbance of their secretory capacity (Fig. (2)) (47).These data directly implicate the EphB4 receptor in the regulation of the patterning of the mammary ductal tree, the epithelial architecture and response to growth controlling signals.

\section{REMODELING OF THE VASCULATURE DURING THE MAMMARY GLAND LIFE CYCLE}

The mammary gland life cycle is exemplified by massive changes in cell number and composition,
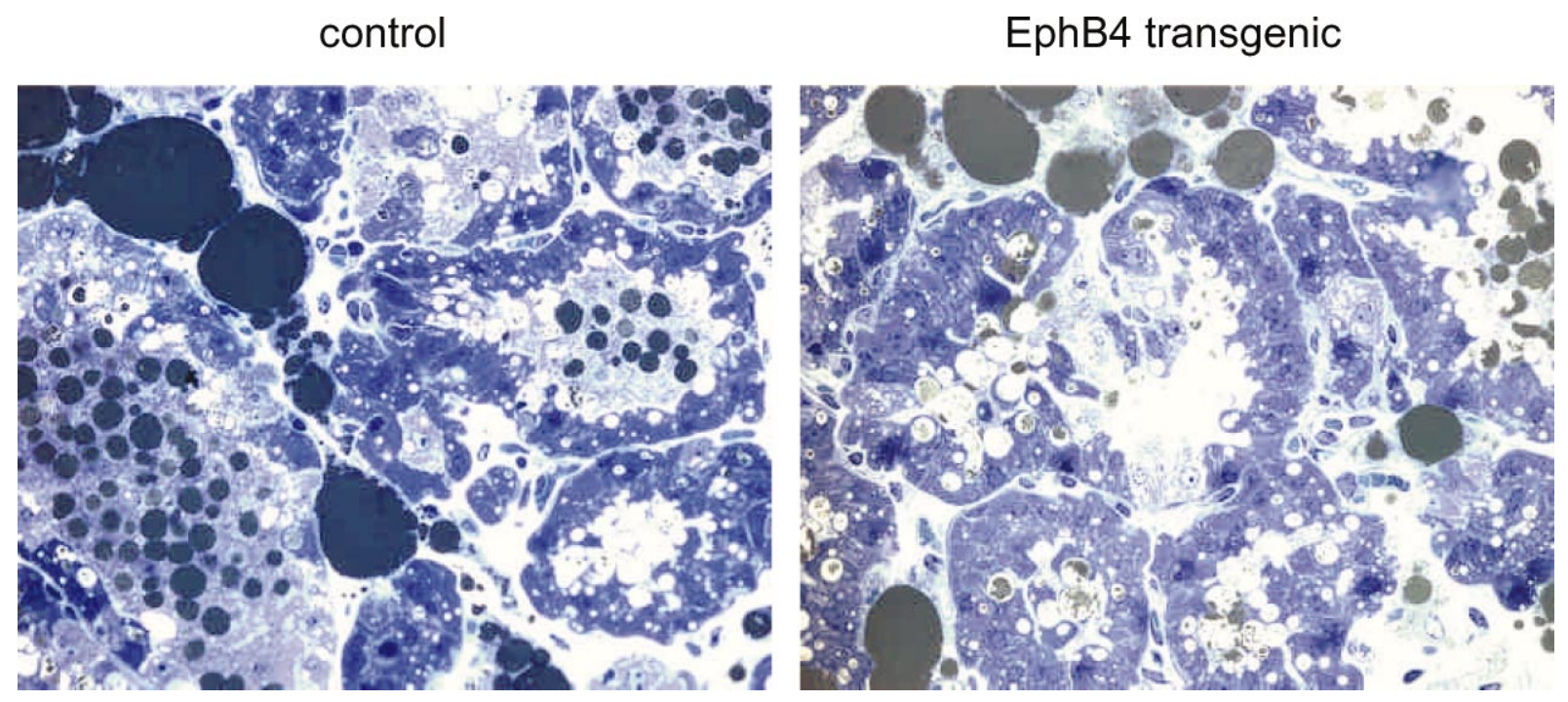

Fig. 2. Semithin sections of lactating mammary glands of control and EphB4 transgenic mice. $1 \mu$ sections were stained with toluene blue. Note the regular appearance of the secretory epithelium in control mammary glands with numerous vesicles reflecting their secretory activity. Dark spots in the lumen indicate secreted milk fat globules. In contrast, the epithelium of EphB4 transgenic mammary glands exhibits a highly irregular structure with many bright and fragile looking cells lacking secretory activity. In addition, clefts between the epithelial cells indicate disturbances in cell-cell interactions. 

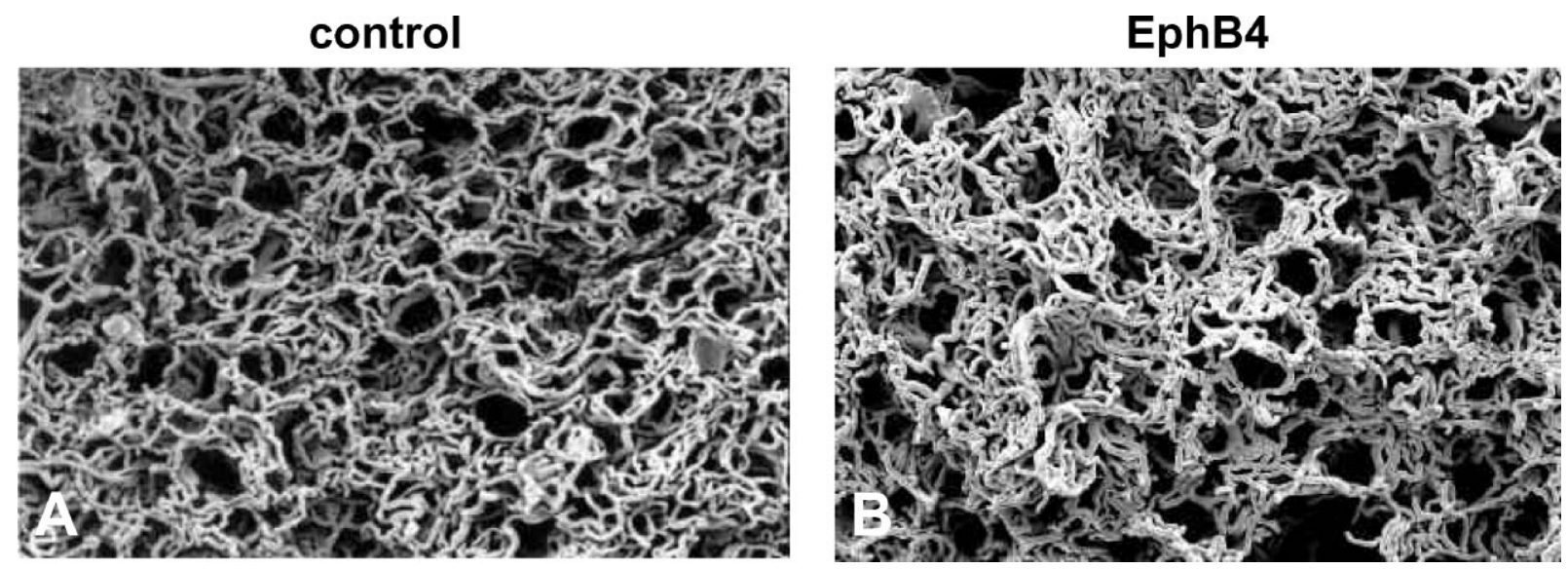

Fig. 3. Scanning electron microscopy photographs of corrosion casts of the mammary gland vasculature at lactation. (A) control; (B) EphB4 transgenic.

architecture, and functionality. These drastic upheavals also involve the mammary endothelial component, which undergoes angiogenic expansion and regression according to physiological demand (48). These events necessitate coordinated communication between the mammary endothelium, the parenchyme, and the stromal components in order to accommodate the changing demands. Indeed, the interaction between mammary endothelial and epithelial cells is not only crucial for vascular development but is also a prerequisite for branching ductal-alveolar morphogenesis (49).

Members of the Eph and ephrin families, notably EphB4 and ephrin-B2, have been implicated not only in the development of the embryonal vasculature but also in neo-vascularization in the adult $(8,10,11)$. In particular, EphB4 and ephrin-B2 appear to mediate critical communication between the arterial and venous endothelia (50). Moreover, nonendothelial expression of EphB receptors has been implicated in endothelial cell guidance (8). In cell culture, stromal expression of EphB4 results in the attenuation of endothelial cells and inhibition of the formation of a vascular network. In contrast, stromal expression of ephrin-B2 results in the stimulation of endothelial cell proliferation and sprouting (51).

Direct evidence for the involvement of the EphB4 receptor in vascular development in the mammary gland has been obtained using transgenic mice expressing the MMTV-LTR-EphB4 transgene. Mercox corrosion cast visualization of the mammary gland vasculature at lactation revealed in control animals a well-developed, highly regular vascular network, each alveolus being surrounded by a honeycomb-like capillary plexus (Fig. 3(A)). In contrast, the vasculature in lactating mammary glands of the EphB4 transgenic animals exhibited a distinctly irregular structure; many of the honeycomb-like structures were poorly discernible, partly collapsed, and aggregated. Furthermore, the capillaries varied considerably in size, and bundles of very thin, disorganized capillaries were observed locally (Fig. 3(B) and unpublished observations). The conclusion that unscheduled expression of EphB4 may interfere with vascular architecture is further supported by observations made in the kidney of these transgenic mice. In addition to the expression in the mammary gland, EphB4 transgene expression was also observed in the epithelial cells constituting the proximal tubules and Bowman's capsule of the kidney. This unscheduled expression correlated with abnormal development of glomerular vasculature and shunt formation reminiscent of aglomerular vascular shunts, a human degenerative glomerulopathy of unknown aetiology (52).

\section{INVOLVEMENT OF Ephs AND EPHRINS IN MAMMARY GLAND CARCINOGENESIS}

Thus far, evidence for the carcinogenic potential of Ephs and ephrins is scarce, and no mutations in human breast cancer have been tightly linked to Eph or ephrin genes (53). The expression of the EphB4 receptor protein has recently been analyzed in human breast cancers (13). In the normal breast EphB4 was expressed in ductal and luminal epithelial cells, 
predominantly during the follicular phase of the estrus cycle. Carcinogenesis was characterized by the loss of EphB4 expression and correlated with tumor progression. Interestingly, in advanced tumors single tumor cells strongly expressing EphB4 were observed in the border region between tumor nodules and the surrounding mesenchyme.

More precise information about the involvement of Eph or ephrin in carcinogenic processes has been obtained from cell culture and transgenic mouse models. Overexpression of the EphA2 protein has been observed in a variety of human breast cancer cell lines, and transfection of normal human breast epithelial cells with EphA2 was sufficient to induce transformation. Interestingly, in both cases the EphA2 molecules remained unphosphorylated, and induction of phosphorylation by exogenous administration of the relevant ephrin-A1 ligand could revert the transformed phenotype $(16,54)$. These data suggest that the Ephs may exert their oncogenic potential in the absence of receptor phosphorylation, in contrast to the oncogenic mechanisms proposed for most receptor tyrosine kinases. Expression of EphA2 and its ligand ephrin-A1 has been demonstrated in the vasculature of human primary breast cancers and of breast-tumor-cell-line-derived tumors in nude mice (55). Thus, EphA2 may be directly involved in tumorinduced angiogenesis, a conclusion supported by the observation that soluble EphA2 receptors are capable of blocking blood vessel recruitment by breast tumor cells (56).

In the EphB4 transgenic mice, EphB4 overexpression per se never gave rise to mammary tumors; however, crossing these animals with MMTV-c-erbB2 transgenic mice led to an approximately $50 \%$ reduction of tumor latency in the double transgenic animals compared to the single transgenic c-erbB2 mice. Moreover, in contrast to the single transgenic c-erbB2 mice, the double transgenic animals rapidly developed metastases to the lungs (47). These data indicate that unscheduled expression of EphB4 can contribute to the establishment of an aggressive tumor phenotype.

These limited experimental data support the concept that Eph and ephrins are not directly involved in the control of cell proliferation and induction of carcinogenesis, but rather contribute to tumor progression and metastasis formation (57). The acquisition of an invasive and metastatic phenotype requires loss of cell contacts to neighboring cells, transient change or loss in integrin-mediated attachment to the basement membrane, invasion into the surround- ing stroma, penetration into the blood stream, and finally extravasation into a permissive tissue, which sustains their further growth. It is conceivable that Eph and ephrin participate at all these levels. Loss of E-cadherin expression, a common feature of almost all carcinomas (58), may impede EphA function and induce EphB expression, allowing migration through the tissue. Moreover, Eph-ephrin signaling can lead to dissociation of focal adhesion points, thereby facilitating the detachment from the basement membrane. Furthermore, Eph and ephrins are instrumental in the formation of a functional vascular network during tumor angiogenesis $(10,11)$. The microvascular density in tumors in turn not only ensures sufficient nutrient supply for growth but also increases the chance of invasive tumor cells entering the blood stream. Finally, the adhesive/repulsive function of Eph and ephrins may facilitate the seeding of tumor cells in a permissive environment.

An alternative possibility, by which Eph and ephrin may contribute to tumor progression, may lie in their postulated involvement in mammary stem cell demarcation, maintenance and differentiation (see above). The classical cancer stem cell hypothesis implicates mutations in this cell population as a potential source of tumorigenic cancer cells. Recent studies have shown that only a small subset of human breast tumor cells is capable of efficiently forming tumors in an immunocompromised mouse model. These cells were characterized by shared cell surface markers reminiscent of a stem cell population (59). Conceivably, modulated Eph and ephrin expression may favor the release, migration, and seeding of initiated mutant stem cells.

\section{CONCLUSIONS}

Members of the Eph and ephrin families, contribute to pattern formation by governing cell adhesion, repulsion, movement, and intermingling. These processes are not only prevalent during embryonic development but also persist in adult organs subjected to continuous tissue dynamics, such as the vasculature, the intestine, the reproductive organs, and the mammary gland. Through their ability to interact not only with each other but also with other signaling pathways and molecules involved in cell-cell contacts or cellmatrix interactions, Ephs and ephrins may participate in different aspects of mammary gland morphogenesis summarized in Fig. 4.

Figure 4, 1\&2: The Eph/ephrin families may be involved in the maintenance, demarcation and control 


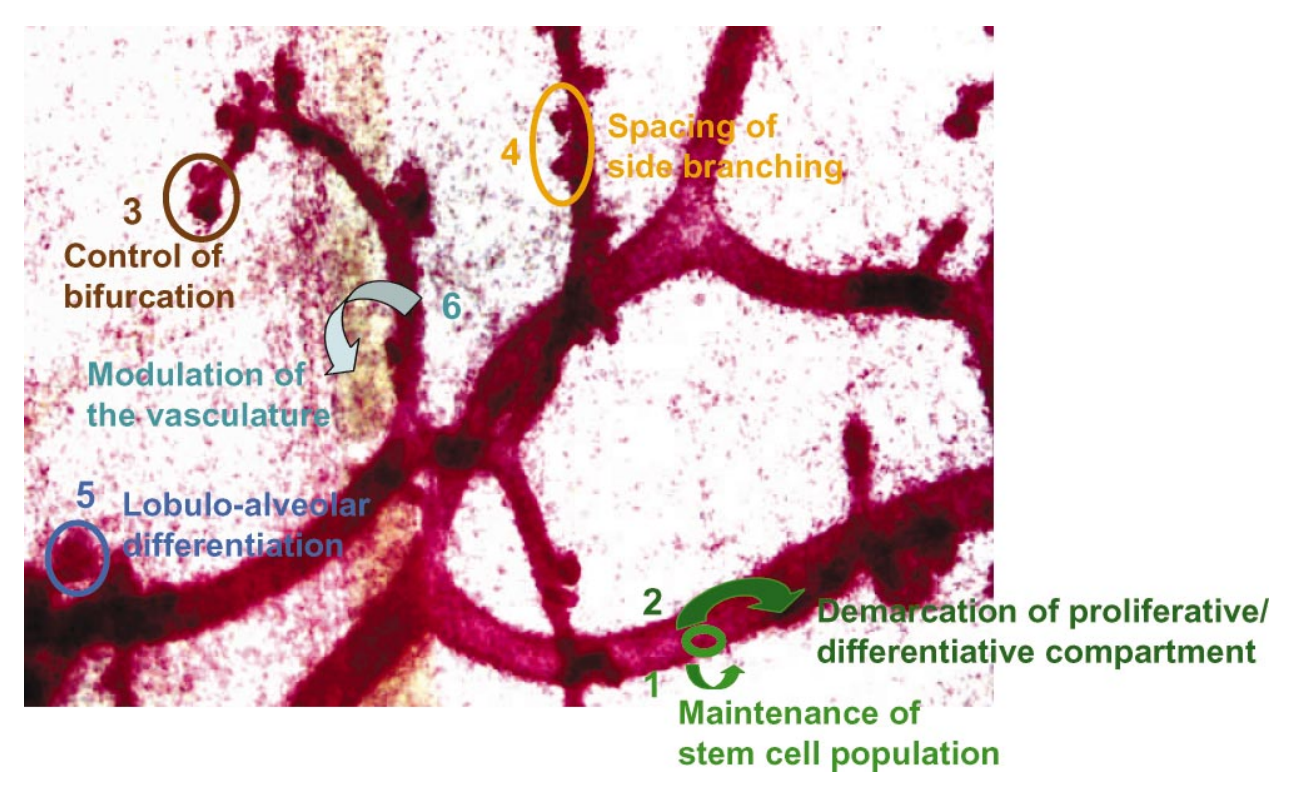

Fig. 4. Schematic representation of the possible functions of Eph receptors and ephrin ligands in mammary gland morphogenesis superimposed on a whole mount preparation of a mammary gland of a 10-week-old virgin mouse. Note the faintly stained blosod vessels in contrast to the densely stained epithelial tree.

of fate of the stem cell population harboring the integrators of positive and negative mammatrophic signals. Many of these signals act in a complex of interepithelial and paracrine interactions (60) in which the Ephs and ephrins may represent additional modulators. This aspect of Eph and ephrin action would not only ensure tissue regeneration and homeostasis but may also have an impact on the susceptibility of the mammary gland to malignant transformation.

Figure 4, 3\&4: The organotypic growth of the mammary parenchyma during puberty involves ductal elongation and morphogenesis as well as pattern formation by bifurcation of the terminal end-buds. Although an understanding of the local paracrine growth control is beginning to emerge (61), the mechanisms responsible for guiding the growing ducts through the fat pads, defining the frequency of bifurcation events, and determining the spacing between the ductal structures are largely unknown. Similarly, the regulation of lobulo-alveolar patterning and number remains unclear. Extrapolated from their known function in guidance of cellular movement during embryogenesis, Ephs and ephrins are probably also instrumental in pattern formation in the pubertal mammary gland and in lobulo-alveolar development during pregnancy. This suggestion is supported by the observation that mammary epithelial over-expression of EphB4 during puberty resulted in growth retardation, reduced bifurcation, and the absence of second- and third-order branching. Moreover, the intensity of budding and lobule formation was diminished by EphB4 expression during pregnancy (47).

Figure 4, 5: Through their ability to modulate cell-cell and cell-matrix interactions, the Ephs and ephrins are also likely to be implicated in the development and maintenance of the alveolar structure at lactation (Fig. 2). The ability to modulate cell adhesion and migration represents an important aspect in the development and maintenance of a functional organ structure in this dynamic organ. This same property, however, may also contribute to tumor progression.

Figure 4, 6: The above suggested roles of Eph receptors and ephrin ligands represent direct modulations of mammary gland morphogenesis. These molecules, however, may also have an indirect influence on growth and functioning of the mammary gland through their capacity to influence the formation of a vascular network. Like every organ, proper functioning of the mammary gland is dependent on an adequate blood supply ensuring sufficient oxygenation and nourishment and delivery of endocrine factors. Any disturbance in the extent and architecture of the vasculature will probably affect the functioning of the other organ components.

In summary, Eph receptors and ephrin ligands are likely to be involved, either alone or in cooperation with other signaling pathways, throughout the entire mammary gland life cycle and are probable 
candidates to fill the gaps in our understanding of the regulatory mechanisms for multiple aspects of mammary development. Their multifaceted functions and signaling complexity represent a major challenge to elucidating their individual contributions to mammary gland development and functioning. Nevertheless, the necessary effort will be worth it and we would like to close this paper by a citation from Cooke and Moens (62): "Eph only it were simple...."

\section{ACKNOWLEDGMENTS}

The authors thank the Swiss National Science Foundation (3100-068104.02) and the Bernese Cancer League for their continuous financial support.

\section{REFERENCES}

1. K. K., Murai and E. B., Pasquale (2003). "Eph"ective signaling: Forward, reverse and cross-talk. J. Cell Sci. 116:2823-2832.

2. K. Kullander and R. Klein (2002). Mechanisms and functions of Eph and ephrin signaling. Nat. Cell Biol. 3:475-486.

3. J. P. Himamen and D. B. Nikolov (2003). Eph signaling: A structural review. Trends Neurosci. 26:46-51.

4. M. Hattori, M. Osterfield, and J. G. Flanagan (2000). Regulated cleavage of a contact-mediated axon repellent. Science 289:1360-1365.

5. A. W. Boyd and M. Lachmann (2001). Signals from Eph and ephrin proteins: A developmental tool kit. Sci. STKE 112:RE20.

6. N. Holder and R. Klein (1999). Eph receptors and ephrins: Effectors of morphogenesis. Development 126:2033-2044.

7. D. G. Wilkinson (2000). Eph receptors and ephrins: Regulators of guidance and assembly. Int. Rev. Cytol. 177-244.

8. R. H. Adams (2002). Vascular patterning by Eph receptor tyrosine kinases and ephrins. Semin. Cell. Dev. Biol. 13:55-60.

9. A.-C. Andres, H. H. Reid, G. Zürcher, R. J. Blaschke, D. Albrecht, and A. Ziemiecki (1994). Expression of two novel eph-related receptor protein tyrosine kinases in mammary gland development. Oncogene 9:235-239.

10. N. W. Gale, P. Baluk, L. Pan, M. Kwan, J. Holash, T. M. DeChiara, D. M. McDonald, and G. D. Yancopoulos (2001). Ephrin-B2 selectively marks arterial vessels and neovascularization sites in the adult, with expression in both endothelial and smooth muscle cells. Dev. Biol. 230:151-160.

11. D. Shin, G. Garcia-Cardena, S.-I. Hayashi, S. Gerety, T. Asahara, G. Stavrakis, J. Isner, J. Folkman, M. A. Gimbrone, and D. J. Anderson (2001). Expression of ephrin-B2 identifies a stable genetic difference between arterial and venous vascular smooth muscle cells as well as endothelial cells, and marks subsets of microvessels at sites of neovascularization. Dev. Biol. 230:139-150.

12. Z. Nikolova, V. Djonov, G. Zürcher, A.-C. Andres, and A. Ziemiecki (1998). Cell type-specific and estrogen dependent expression of the receptor tyrosine kinase EphB4 and ist ligand ephrin-B2 during mammary gland morphogenesis. J. Cell. Sci. 111:2741-2751.
13. G. Berclaz, H. J. Altermatt, V. Rohrbach, B. Flütsch, V. Djonov, A. Ziemiecki, E. Dreher, and A.-C. Andres (2002). Loss of EphB4 tyrosine kinase expression during carcinogenesis of the human breast. Oncol. Rep. 9:25-30.

14. P. A. Brittis, Q. Lu, and J. G. Flanagan (2002). Axonal protein sythesis provides a mechanism for localized regulation at an intermediate target. Cell 110:223-235.

15. G. Berclaz, E. Karamitopoulou, L. Mazzucchelli, V. Rohrbach, E. Dreher, A. Ziemiecki, and A.-C. Andres (2003). Activation of the receptor tyrosine kinase EphB4 in endometrial hyperplasia and endometrial carcinoma. Ann. Oncol. 14:220-226.

16. D. P. Zelinski, N. D. Zantek, J. C. Stewart, A. R. Irizarry, and M. S. Kinch (2001). EphA2 overexression causes tumorigenesis of mammary epithelial cells. Cancer Res. 61:2301-2306.

17. D. P. Zelinski, N. D. Zantek, J. Walker-Daniels, M. A. Peters, E. J. Taparowsky, and M. S. Kinch (2002). Estrogen and myk negatively regulate expression of the EphA2 tyrosine kinase. $J$. Cell. Biochem. 85:714-720.

18. N. Barker, G. Huls, V. Korinek, and H. Clevers (1999). Restricted high level expression of Tcf-4 protein in intestinal mammary epithelium. Am. J. Pathol. 154:29-35.

19. E. Batlle, J. T. Henderson, H. Beghte, M. W. M. van den Born, E. Sancho, G. Huls, J. Meeldijk, J. Robertson, M. van de Wetering, T. Pawson, and H. Clevers (2002). $\beta$-Catenin and TCF mediate cell positioning in the intestinal epithelium by controlling the expression of EphB/ephrinB. Cell 111:251-263.

20. C. A. Cowan and M. Henkemeyer (2002). Ephrins in reverse, park and drive. Trends Cell Biol. 12:339-346.

21. G. B. Silberstein (2001). Postnatal mammary gland morphogenesis. Microsc. Res. Tech. 52:155-163.

22. C. W. Daniel, P. Strickland, and Y. Friedmann (1995). Expression and functional role of E- and p-caherins in mouse mammary ductal morphogenesis and growth. Dev. Biol. 169:511519.

23. S. Orsulic and R. Kemler (2000). Expression of Eph receptors and ephrins is differentially regulated by E-cadherin. $J$. Cell. Sci. 113:1793-1802.

24. N. D. Zantek, M. Azimi, M. Fedor-Chaiken, B. Wang, R. Brackenbury, and M. S. Kinch (1999). E-cadherin regulates the function of the EphA2 receptor tyrosine kinase. Cell Growth Diff. 10:629-638.

25. A. B. Zhadanov, D. W. Provance, C. A. Speer, J. D. Coffin, D. Goss, J. A. Blixt, C. M. Reichert, and J. A. Mercer (1999). Absence of the tight junctional protein AF-6 disrupts epithelial cell:cell junctions and cell polarity during mouse development. Curr. Biol. 26:880-888.

26. B. Hock, B. Bohme, T. Karn, T. Jamamoto, K. Kaibuchi, U. Holtrich, S. Holland, T. Pawson, H. Rubsamen-Waigean, and K. Strebharot (1998). PDZ-domain-mediated interaction of the Eph-related receptor tyrosine kinase EphB3 and the Rasbindingt protein AF-6 depend on the kinase activity of the receptor. Proc. Natl. Acad. Sci. U. S. A. 95:9779-9784.

27. T. Linnemann, M. Geyer, B. K. Jaitner, C. Block, H. R. Kalbitzer, A. Wittinghofer, and C. Herrmann (1999). Thermodynamic and kinetic characterization of the interaction between the Ras binding domain of AF6 and members of the subfamily. J. Biol. Chem. 274:13556-13562.

28. R. M. Katso, R. B. Russell, and T. S. Ganesan (1999). Functional analysis of H-ryk, an atypical member of the receptor tyrosine kinase family. Mol. Cell. Biol. 19:6427-6440.

29. A.-C. Andres, G. Zürcher, V. Djonov, M. Flück, and A. Ziemiecki (1995). Protein tyrosine kinase expression during 
the estrous cycle and carcinogenesis of the mammary gland. Int. J. Cancer 63:288-296.

30. C. M. Hovens, S. A. Staker, A.-C. Andres, A. G. Harpur, A. Ziemiecki, and A. F. Wilks (1992). RYK, a receptor tyrosine kinase-related molecule with unusual kinase domain motifs. Proc. Natl. Acad. Sci. U.S.A. 89:11818-11822.

31. E. Trivier and T. S. Ganesan (2002). Ryk, a catalytically inactive receptor tyrosine kinase associates with EphB2 and EphB3 but does not interact with AF-6. J. Biol. Chem. 277:23037-23043.

32. M. M. Halford, J. Armes, M. Buchert, V. Meskenaite, D. Grail, M. L. Hibbs, A. F. Wilus, P. G. Parlie, D. F. Newgreen, C. M. Hovens, and S. A. Stacuer (2000). Ryk deficient mice exhibit craniofacial defects associated with perturbed Eph receptor crosstalk. Nat. Genet. 25:414-418

33. C. H. Streuli and G. M. Edwards (1998). Control of normal mammary epithelial phenotype by integrins. J. Mamm. Gland Biol. Neoplasia 3:151-163.

34. K. Nagashima, A. Endo, H. Ogita, A. Kawana, A. Vamagishi, A. Kitabatake, M. Matsuda, and N. Mochizuki (2002). Adaptor protein Crk is required for ephrin-B1 induced membrane ruffling and focal complex assembly of human aortic endothelial cells. Mol. Biol. Cell. 13:4231-4242.

35. H. Miao, E. Burnett, M. Kinch, E. Simon, and B. Wang (2000). Activation of the EphA2 kinase suppresses integrin function and causes focal adhesion-kinase dephosphrylation. Nat. Cell Biol. 2:62-69.

36. J. X. Zou, B. Wang, M. S. Kalo, A. H. Zisch, E. B. Pasquale, and E. Ruoslahti (1999). An Eph receptor regulates integrin activity through R-ras. Proc. Natl. Acad. Sci. U.S.A. 96:13813-13818.

37. U. Huynh-Do, C. Vindis, H. Liu, D. P. Cerretti, J. T. Mc Grew, M. Enriquez, J. Chen, and T. O. Daniel (1999). Ephrin-B1 transduces signals to activate integrin-mediated migration, attachment and angiogenesis. J. Cell Sci. 115:3073-3081.

38. J. J. Steinle, C. J. Meininger, R. Forough, G. Wu, M. H. Wu, and H. J. Granger (2002). EphB4 receptor signaling mediates endothelial cell migration and proliferation via the phsphatidylinositol 3-kinase pathway. J. Biol. Chem. 277:43830-43835.

39. C. Gu and S. Park (2001). The ephA 8 receptor regulates integrin activity through $\mathrm{p} 110$ (gamma) phosphatidylinositol-3 kinase in a tyrosine kinase activity-independent manner. Mol. Cell Biol. 21:4579-4597.

40. A. Pandey, D. F. Lazar, A. R. Saltiel, and V. M. Dixit (1994). Activation of the Eck receptor tyrosine kinase stimulates phosphatidylinositol 3-kinase activity. J. Biol. Chem. 269:3015430157.

41. A. Palmer, M. Zimmer, K. S. Erdmann, V. Eulenburg, A. Porthin, R. Heumann, U. Deutsch, and R. Klein (2002). EphrinB2 phosphorylation and reverse signaling: Regulation by src kinases and PTP-BL phosphatase. Mol. Cell 9:725-737.

42. M. Bienz and H. Clevers (2000). Linking colorectal cancer to Wnt signaling. Cell 103:311-320.

43. S. J. Weber-Hall, D. J. Phippard, C. C. Niemeyer, and T. C. Dale (1994). Developmental and hormonal regulation of Wnt gene expression in the mouse mammary gland. Differentiation 57:205-214.

44. C. Brisken, A. Heineman, T. Chavarria, B. Elenbaas, J. Tan, S. K. Dey, J. A. McMahon, A. P. McMahon, and R. A. Weinberg (2000). Essential function of Wnt-4 in mammary gland development downstream of progesterone signaling. Genes Dev. 14:650-654.

45. G. H. Smith and G. Chepko. (2001). Mammary epithelial stem cells. Microsc. Res. Techn. 52:190-204.
46. L. Alonso and E. Fuchs (2003). Stem cells in the skin: Waste not, wnt not. Genes Dev 17:1189-1200

47. N. Munarini, R. Jaeger, S. Abderhalden, G. Zürcher, V. Rohrbach, S. Lörcher, B. Pfanner-Meyer, A.-C. Andres, and A. Ziemiecui (2002). Altered mammary epithelial development, pattern formation and involution in transgenic mice expressing the EphB4 receptor tyrosine kinase. J. Cell Sci. 115:25-37.

48. V. Djonov, A.-C. Andres, and A. Ziemiecki (2001). Vascular remodeling during the normal and malignant life cycle of the mammary gland. Microsc Res. Tech. 52:182-189.

49. M. P. Shekar, J. Werdell, and L. Tait (2000). Interaction with endothelial cells is a prerequisite for branching ductal-alveolar morphogenesis and hyperplastic human breast epithelial cells: Regulation by estrogens. Cancer Res. 60:439-449.

50. R. H. Adams (2003). Molecular control of arterial-venous blood vessel identity. J. Anat. 202:105-112.

51. R. H. Adams, G. A. Wilkinson, C. Weiss, F. Diella, N. W. Gale, U. Deutsch, W. Risan, and R. Klein (1999). Roles of ephrinB ligands and EphB receptors in cardiovascular development: Demarcation of arterial/venous domains, vascular morphogenesis and sprouting angiogenesis. Genes Dev. 13:295-306.

52. A.-C. Andres, N. Munarini, V. Djonov, S. Bruneau, G. Zürcher, S. Lörcher, V. Rohrbach, and A. Ziemiecki (2003). EphB4 receptor tyrosine kinase transgenic mice develop glomerulopathies reminiscent of aglomerular vascular shunts. Mech. Dev. 120:511-516.

53. M. Nakamoto and A. D. Bergemann (2002). Diverse roles for the Eph family of receptor tyrosine kinases in carcinogenesis. Microsc. Res. Tech. 59:58-67.

54. N. D. Zantek, J. Walker-Daniels, J. Stewart, R. K. Hansen, D. Robinson, H. Miao, B. Wang, H. J. Kung, M. J. Bissell, and M. S. Kinch (2001). MCF-10A-NeoST: A new cell system for studying cell-ECM and cell-cell interactions in breast cancer. Clin. Cancer Res. 7:3640-3648.

55. K. Ogawa, R. Pasqualini, R. A. Lindberg, R. Kain, A. R. Freeman, and E. B. Pasquale (2000). The ephrin-A1 ligand and its receptor, EphA2, are expressed during tumor neovascularization. Oncogene 19:6043-6052.

56. D. M. Brantley, N. Cheng, E. J. Thompson, Q. Lin, R. A. Brekken, P. E. Thrope, R. S. Muraoka, D. P. Cerretti, A. Pozzi, D. Jackson, C. Lin, and J. Chen (2002). Soluble EphA receptors inhibit tumor angiogenesis and progression in vivo. Oncogene 21:7011-7026.

57. R. Klein (2001). Excitatory Eph receptors and adhesive ephrin ligands. Curr. Opin. Cell Biol. 13:196-203.

58. G. Christofori and H. Semb (1999). The role of the cell-adhesion molecule E-cadherin as a tumor suppressor gene. Trends Biol. Sci. 24:73-76

59. M. Al-Hajj, M. S. Wicha, A. Benito-Hernandez, S. J. Morrison, and M. F. Clarke (2003). Prospective identification of tumorigenic breast cancer cells. Proc. Natl. Acad. Sci. U.S.A. 100:39833988

60. C. Brisken, S. Park, T. Vass, J. P. Lyndon, B. W. O'Malley, and R. A. Weinberg (1998). A paracrine role for the epithelial progesterone receptor in mammary development. Proc. Natl. Acad. Sci. U.S.A. 95:5076-5081.

61. G. B. Silberstein, (2001). Tumor-stromal interactions. Role of the stroma in mammary gland development. Breast Cancer Res. 3:218-223.

62. J. E. Cooke and C. B. Moens (2002). Boundary formation in the hindbrain: Eph only it were simple. . . Trends Neurosci. 25:260267. 